\title{
AVALIAÇÃO SENSORIAL DO MEL DE CINCO DIFERENTES ORIGENS FLORAIS
}

Vilson Matias Pinto1, Gabriella Castro Cunha ${ }^{1}$, Alexsandra Valéria Sousa Costa de Lima ${ }^{1}$, Márcio Ramatiz Lima dos Santos ${ }^{1}$

'Instituto Federal Goiano - Campus Ceres, Ceres, GO. E-mail: vimatiashti@hotmail.com

\section{RESUMO}

Objetivou-se conhecer o perfil do consumidor e avaliar a aceitação, intenção de compra e preferência do mel de cinco diferentes origens florais. Os testes de aceitação por atributos (aroma, sabor, aparência, viscosidade, impressão global) foram avaliados mediante escala estruturada de nove pontos e os de intenção de compra com escala estruturada de cinco pontos. Os resultados do perfil de consumidores foram avaliados em porcentagem. Os resultados de aceitação e intenção de compra foram submetidos à análise de variância e as diferenças das médias comparadas por teste de Tukey ao nível de $5 \%$ de significância. Os resultados de ordenaçãopreferência foram analisados através do teste de Friedman. A maioria dos provadores: são do sexo feminino (59\%); consome mel (79\%); não sabe diferenciar o mel tradicional do orgânico (64\%); compraria mel orgânico (89\%); conhece (67\%), consome (75\%) e compraria (78\%) mel do produtor local; prefere comprar (67\%) e consumir (72\%) mel com selo do Serviço de Inspeção Federal. A maioria dos consumidores tem conhecimento sobre o mel, porém não costumam ler os rótulos dos produtos. Independente do tipo de florada todas as amostras de mel tiveram uma boa aceitação $(7,04$ a 7,86$)$ para todos os parâmetros avaliados e boa intenção de compra $(3,76$ a 4,14). O mel de florada silvestre foi mais preferido quando comparado aos de floradas de laranjeira, do sertão e de cipó de uva. Independente do tipo de florada todas as amostras obtiveram índice de aceitabilidade (IA) acima de $70 \%$. Portanto todas as amostras serão aceitas pelo público consumidor.

Palavras-chave: apicultura; Apis mellifera; legislação; qualidade.

\section{SENSORY EVALUATION OF FIVE DIFFERENT FLORAL ORIGINS HONEY}

\section{ABSTRACT}

The objective was to know the profile of the consumer and to evaluate the acceptance, intention of purchase and preference of the honey of five different floral origins. The attributes acceptance tests (aroma, taste, appearance, viscosity, overall impression) were evaluated using a structured nine-point scale and those with a five-point structured scale. The results of the consumer profile were evaluated in percentage. The results of acceptance and intention to purchase were submitted to analysis of variance and the differences of the means compared by Tukey test at the level of $5 \%$ of significance. The order-preference results were analyzed using the Friedman test. The majority of the tasters: are female (59\%); Consumes honey $(79 \%)$; Does not know how to differentiate traditional honey from organic (64\%); Buy organic honey (89\%); Knows (67\%), consumes (75\%) and will buy (78\%) honey from the local producer; Prefers to buy (67\%) and consume (72\%) honey with seal of the Federal Inspection Service. Most consumers are knowledgeable about honey, but do not usually read product labels. Regardless of the flowering type, all honey samples had a good acceptance (7.04 to 7.86) for all parameters evaluated and good intention to buy (3.76 to 4.14). Honey from wildflowers was more preferred when compared to orange blossom, sertão and grapevine. Regardless of the type of flowering, all the samples had 
an acceptability index (IA) above 70\%. Therefore all samples will be accepted by the consumer public.

Keywords: beekeeping; Apis mellifera; legislation; quality.

\section{INTRODUÇÃO}

A criação racional de abelhas constitui-se de uma atividade em que se consegue obter bons resultados econômicos, ecológicos e sociais. Essa atividade, desenvolvida ao longo do tempo por pequenos, médios e grandes produtores, vem despertando o interesse de muitos criadores e instituições do Brasil (RODRIGUES et al., 2005).

O mel, por definição, é um produto natural de abelhas obtido a partir do néctar das flores (mel floral), de secreções de partes vivas das plantas ou de excreções de insetos sugadores de partes vivas das plantas (mel de melato). Durante a sua elaboração, há interferência de variáveis não controladas pelo homem, como clima, floração, presença de insetos sugadores e outros fatores (CAMPOS et al., 2003).

Existem dezenas de variedades de méis que podem ser diferenciadas pela flora, pela localidade (ou região) ou época de colheita, ou ainda, segundo as técnicas de preparação. Rigorosamente, não existe mel monofloral, contudo, uma pequena quantidade de néctar de outras plantas melíferas não influi marcadamente no seu sabor e na sua cor onde predomine o néctar de uma única espécie de flores (BASTOS, 2002). Segundo Souza et al. (2012), o Brasil possui cerca de 350 mil apicultores e 700 entidades entre associações, cooperativas e núcleos produtivos. Atualmente, o maior estado produtor de mel é o Rio Grande do Sul, que produz sete mil toneladas por ano.

O controle de qualidade é uma etapa importante para que o produto seja comercializado com as suas principais características, que facilitam sua utilização e tem uma adequada apresentação e conservação (LACERDA et al., 2010).

Os principais parâmetros sensoriais utilizados para avaliar a qualidade do mel são: a cor, o aroma, o sabor, a fluidez, cristalização e a interação entre diferentes componentes, características extremamente importantes para agregar valor ao produto (IMPERATRIZ-FONSECA et al. 2006; SODRÉ et al., 2006).

A análise sensorial de mel é uma ferramenta muito importante para avaliação da sua qualidade. No Brasil, ainda não há tradição na utilização desta ferramenta, enquanto na Europa há escolas que formam profissionais capazes de identificar a origem botânica através da análise sensorial. As características sensoriais também determinam o valor do produto. A análise sensorial pode ser realizada por métodos que visam avaliar diferenças entre produtos (como os métodos descritivos) ou por métodos que visam avaliar aceitação e/ou preferência (testes afetivos) (FERREIRA et al., 2009).

Diante destes contextos relatados, torna-se oportuno conhecer o perfil do consumidor e avaliar a aceitação, intenção de compra e preferência do mel de cinco diferentes origens florais.

\section{MATERIAL E MÉTODOS}

As amostras de mel foram obtidas em supermercados no município de Goiânia - Goiás, com as respectivas floradas: flores silvestres, flores de eucalipto, flores de laranjeiras, flores do sertão e flores de cipó de uva, todas da mesma marca. As análises sensoriais foram realizadas no Instituto Federal Goiano (IF Goiano) Campus Ceres, com a participação de cinquenta provadores não treinados, sendo alunos e servidores do Campus. Foi realizado o teste cego, ou seja, a marca das amostras de mel e os tipos de floradas não foram informadas aos provadores para que não 
houvesse interferência nos resultados. A todos os provadores foi aplicado o Termo de Consentimento Livre e Esclarecido para participarem da análise sensorial.

Durante as avaliações sensoriais foi aplicado aos provadores das amostras de mel um questionário estruturado contendo informações sobre sexo (feminino e masculino), faixa etária, hábito de consumir mel (sim ou não), frequência de consumo de mel (todos os dias; duas vezes na semana; uma vez na semana; quinze em quinze dias; ou uma vez por mês), tipo de mel que mais consome (orgânico, produtor local, flora silvestre, selo do SIF, outro, ou não consome mel), costume sobre ler os rótulos dos méis que consome (sempre, com frequência, ás vezes, ocasionalmente, ou nunca consome mel), além de diversas outras características relacionadas ao consumo, à obtenção do produto e o conhecimento sobre o Serviço de Inspeção Federal.

Foi realizado o teste de aceitação em que a cada provador foram oferecidas de forma monádica as cinco amostras, servidas com biscoitos de água em sal, em pratos plásticos descartáveis de cor branca, codificados com números de três dígitos. Os julgadores avaliaram as amostras, mediante escala estruturada de nove pontos, variando de 1 (desgostei muitíssimo) a 9 (gostei muitíssimo), segundo MINIM (2010), avaliando-se os parâmetros aroma, sabor, aparência, viscosidade e impressão global.

Determinou-se o Índice de Aceitabilidade (IA), adotando-se a fórmula de Teixeira et al. (1987), em que o IA com valor igual ou maior que 70\% representa boa aceitabilidade do produto: $\mathrm{IA}(\%)=\mathrm{A} \times 100 / \mathrm{B}$, em que, $\mathrm{A}=$ nota média obtida para o produto e $\mathrm{B}=$ nota máxima da escala.

Foi avaliada a intenção de compra das amostras de mel aplicando-se uma escala estruturada de cinco pontos, perguntando-se aos provadores se certamente comprariam ou não comprariam os produtos caso estivessem disponíveis no mercado, utilizando-se uma escala entre 1 (certamente compraria) e 5 (certamente não compraria).

As amostras de mel também foram analisadas pelo teste de ordenação-preferência, em que a cada provador foram oferecidas, simultaneamente, as cinco amostras, em temperatura ambiente e servidas nas cabines individuais, iluminadas com luz branca, sendo solicitado aos provadores que ordenassem as amostras em ordem crescente de preferência atribuindo 1 (menor preferência) até 5 (maior preferência), respectivamente.

Os resultados do perfil de consumidores foram avaliados em porcentagem e apresentados na forma de histogramas. Os resultados do índice de aceitabilidade foram apresentados em porcentagem. Em relação aos testes de aceitação e intenção de compra os resultados foram submetidos à análise de variância e as diferenças das médias comparadas por teste de Tukey ao nível de 5\% de significância, utilizando-se o software $R$ versão 2.11.1 (R DEVELOPMENT CORE TEAM, 2010). Os resultados das análises sensoriais de ordenação-preferência foram analisados através do teste de Friedman, (Tabela de NEWELL e MAC FARLANE), segundo Minim (2010). Foi utilizado o delineamento experimental inteiramente casualizado, com três repetições de processamento/três lotes, baseado na disponibilidade das amostras nos diferentes pontos de venda selecionados, em cada dia em que foram efetuadas as amostragens, e nas datas de fabricação dos diferentes produtos disponíveis em cada ponto de venda (ROCHA et al., 2006). Preferencialmente, foram selecionadas as amostras com data de fabricação mais recente (BARROS NETO et al., 2003).

\section{RESULTADOS E DISCUSSÃO}

As maiorias dos provadores que responderam o questionário são do sexo feminino (59\%), como faixa etária de idade presente entre < 18 até 29 anos, como grau de instrução de ensino médio incompleto (37\%), sendo que maior parte dos consumidores tem o habito de consumir mel (79\%) e com frequência de consumo em uma vez por mês (Figura 1). 
Figura 1. Informações básicas dos provadores.

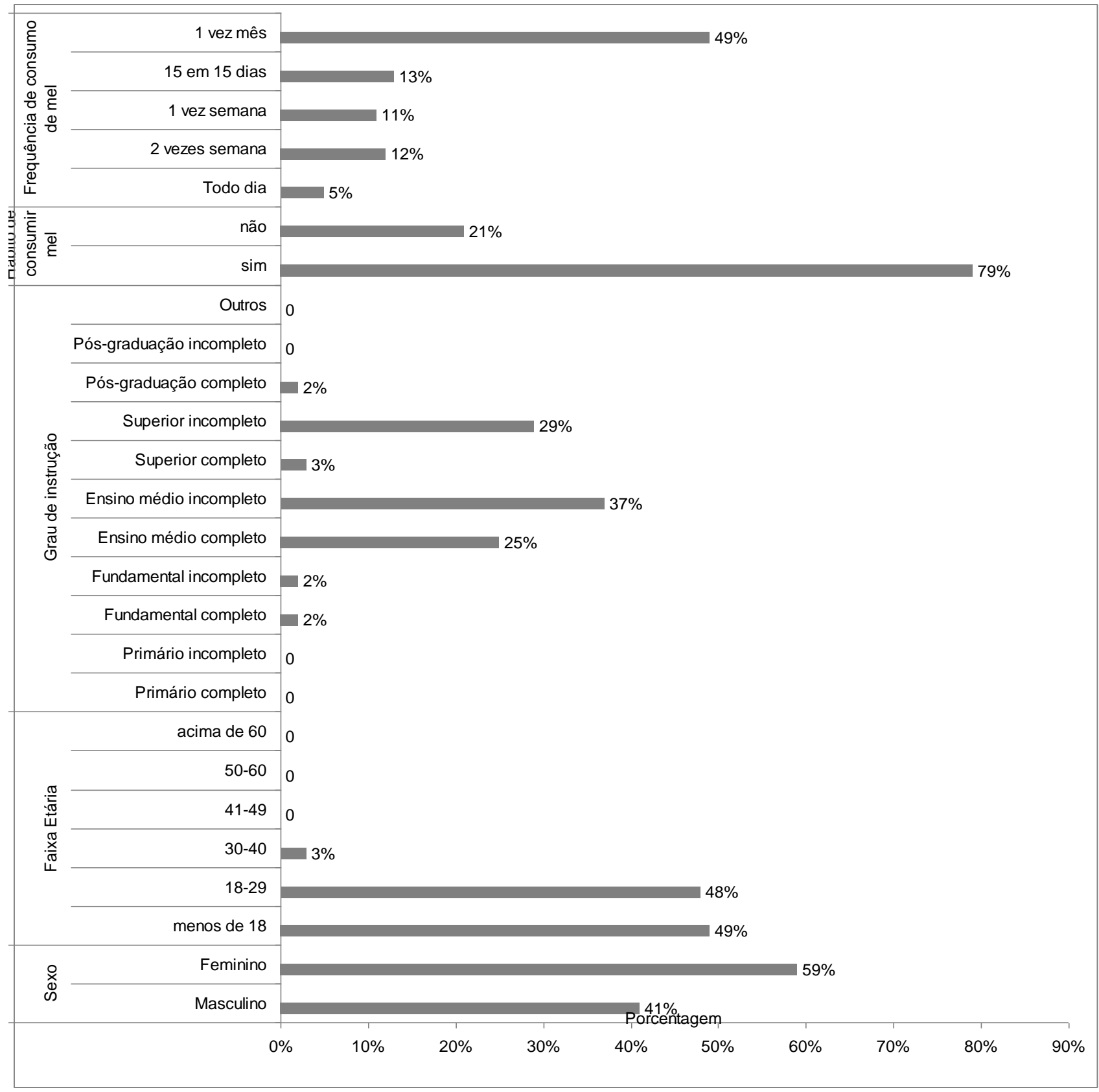


Observou-se que o mel mais consumido (Figura 2) é o do produtor rural (58\%), os consumidores responderam que nunca (42\%) costumam ler os rótulos dos méis que compram, entretanto $39 \%$ das pessoas observam no rótulo o prazo de validade do produto. O conhecimento sobre o mel orgânico é de $51 \%$ dos provadores, porém aqueles que não sabem diferenciar o mel tradicional do orgânico representa $64 \%$.

Figura 2. Informações sobre conhecimento geral do mel

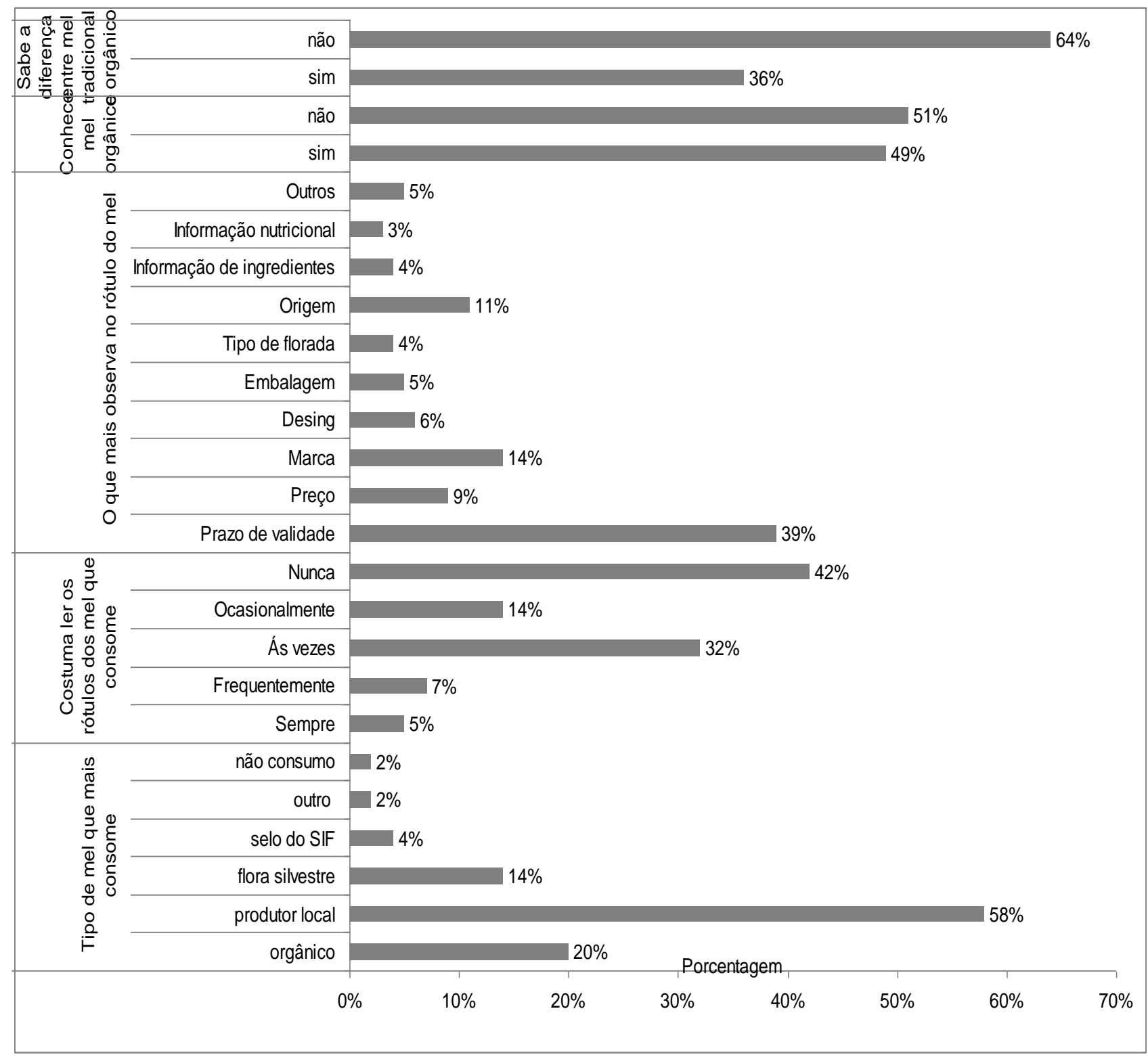


O consumo de mel orgânico é de $56 \%$ e o número de pessoas que comprariam o mesmo é grande (89\%). Constatou-se que $73 \%$ preferem consumir o mel tradicional, sendo que $75 \%$ dos provadores consomem o mel do produtor local e $67 \%$ conhecem os méis (Figura 3).

Figura 3. Informações de consumo de mel.

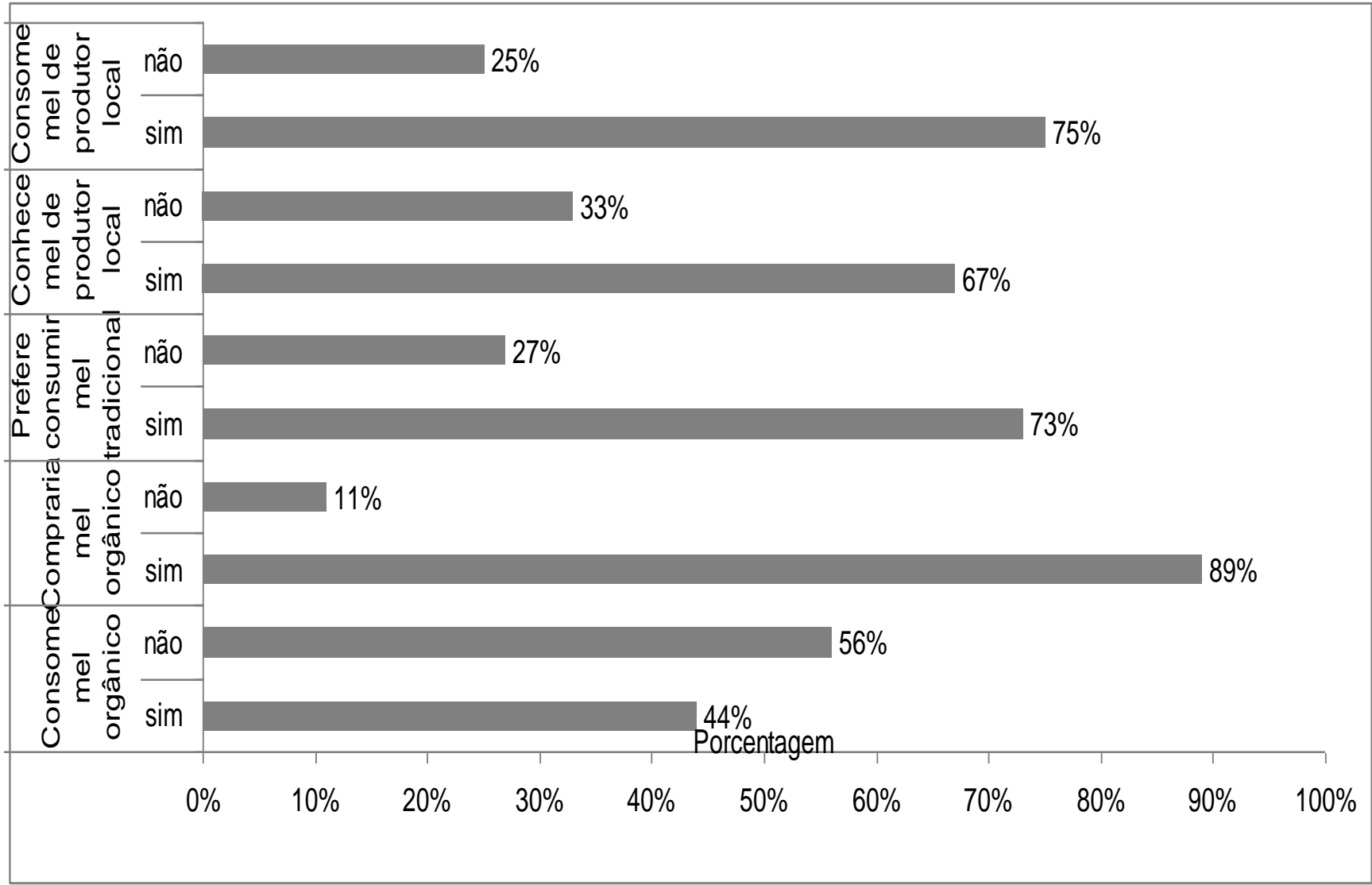

A porcentagem de pessoas que comprariam o mel de produtor local (Figura 4) é de 78\%. Constatou-se que $54 \%$ dos consumidores não conhecem mel com selo de Serviço de Inspeção Federal (SIF). Mesmo não conhecendo 67 \% preferem comprar e $72 \%$ consumir o mel com selo do SIF. Os provadores ás vezes (35\%) costumam ler os rótulos dos produtos que consomem, o que é mais observado no rótulo é o prazo de validade (48\%). 
Figura 4. Informações sobre qualidade do produto de consumo.

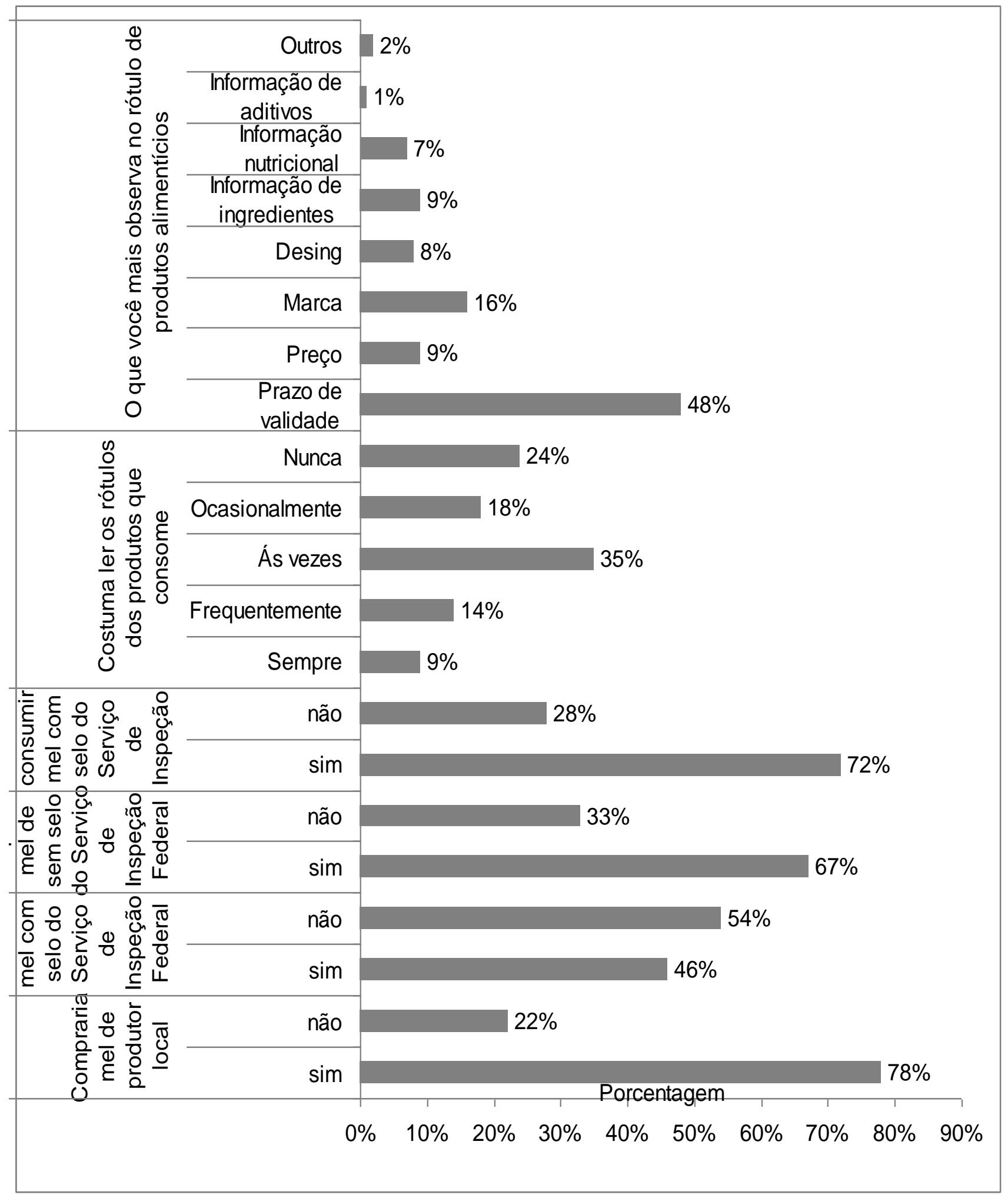


Com base nos resultados obtidos (Tabela 1 ) observou-se que não houve diferença significativa entre os tratamentos (T1, T2, T3, T4 e T5), os quais foram iguais entre si quanto à intenção de compra. Constatou-se que independente do tipo de florada os provadores provavelmente comprariam as amostras. Nenhuma das cinco amostras avaliadas obteve resultado negativo, ou seja, em que os avaliadores certamente não comprariam o produto (Tabela 1). Teixeira et al. (1987), concluíram que o aumento da aceitabilidade se deve à relação entre os estímulos da visão, gustação e olfação. Determinando assim a avaliação de um alimento e sua aceitabilidade no mercado consumidor.

Tabela 1. Valores médios de intenção de compra de mel com marca igual e floradas diferentes.

\begin{tabular}{cc}
\hline Tratamentos & Intenção de compra \\
\hline T1 & $4,10 \mathrm{a}$ \\
T2 & $3,96 \mathrm{a}$ \\
T3 & $3,76 \mathrm{a}$ \\
T4 & $4,14 \mathrm{a}$ \\
T5 & $4,02 \mathrm{a}$ \\
\hline
\end{tabular}

Legenda: $\mathrm{T} 1$ = flores silvestre; $\mathrm{T} 2=$ flores de eucalipto; $\mathrm{T} 3=$ flores de laranjeira; $\mathrm{T} 4=$ flores do sertão; $\mathrm{T} 5=$ flores de cipó de uva.

Médias na mesma coluna e com letras iguais não diferem $(p \leq 0,05)$ significativamente entre si, pelo teste de Tukey.

Através dos resultados obtidos (Tabela 2) para os atributos de impressão global, aroma, sabor, aparência e viscosidade constatou-se que todos os tratamentos foram significativamente iguais e obteve-se a aceitação entre 7,04 e 7,86 ) (>80\%), em que os provadores gostaram moderadamente em todos os aspectos, para todas as floradas avaliadas. Todas as cinco amostras foram aceitas pelos consumidores obtendo-se assim bons resultados para os diferentes méis avaliados (Tabela 2).

Tabela 2. Valores médios de aceitação por atributos das amostras de mel com marca igual e floradas diferentes.

\begin{tabular}{cccccc}
\hline Tratamentos & Impressão global & Aroma & Sabor & Aparência & Viscosidade \\
\hline T1 & $7,36 a$ & $7,30 a$ & $7,68 a$ & $7,36 a$ & $7,48 a$ \\
T2 & $7,86 a$ & $7,44 a$ & $7,62 a$ & $7,78 a$ & $7,52 a$ \\
T3 & $7,42 a$ & $7,04 a$ & $7,32 a$ & $7,26 a$ & $7,08 a$ \\
T4 & $7,32 a$ & $7,44 a$ & $7,58 a$ & $7,66 a$ & $7,24 a$ \\
T5 & $7,82 a$ & $7,38 a$ & 7,42 & $7,74 a$ & $7,76 a$ \\
\hline
\end{tabular}

Legenda: $\mathrm{T} 1$ = flores silvestre; T2= flores de eucalipto; T3= flores de laranjeira; T4= flores do sertão; T5= flores de cipó de uva.

Médias na mesma coluna e com letras iguais não diferem $(p \leq 0,05)$ significativamente entre si, pelo teste de Tukey.

De acordo com Santos et al. (2011), nas análises dos dados observou-se que o mel dos municípios de Santo Estevão, Santa Bárbara e Irará obtiveram maiores médias para aceitabilidade, sendo estatisticamente superiores aos das demais amostras analisadas no estado da Bahia. Segundo Guyton (2006), a cor de um alimento é um dos parâmetros que influencia fortemente na caracterização da qualidade sensorial e na aceitabilidade de produtos, obtendo uma ótima intensão de compra.

Independente do tipo de florada todas as amostras (Tabela 3) obtiveram índice de aceitabilidade (IA) acima de 70\%. Segundo Teixeira et al. (1987) o IA maior ou igual a 70\% indica que o produto teve boa aceitação e pode ser colocado no mercado para comercialização. Portanto todas as amostras serão aceitas pelo público consumidor. 
Tabela 3. Índice de aceitabilidade (\%) das amostras de mel com marca igual e floradas diferentes.

\begin{tabular}{cccccc}
\hline Tratamentos & Impressão global & Aroma & Sabor & Aparência & Viscosidade \\
\hline T1 & 82 & 81 & 85 & 82 & 83 \\
T2 & 87 & 83 & 85 & 86 & 84 \\
T3 & 82 & 78 & 81 & 81 & 79 \\
T4 & 81 & 83 & 84 & 85 & 80 \\
T5 & 87 & 82 & 82 & 86 & 86 \\
\hline
\end{tabular}

Legenda: $\mathrm{T} 1$ = flores silvestre; $\mathrm{T} 2=$ flores de eucalipto; $\mathrm{T} 3=$ flores de laranjeira; $\mathrm{T} 4=$ flores do sertão; $\mathrm{T} 5=$ flores de cipó de uva.

Em relação à preferência (Tabela 4) dos méis em função das floradas não houve diferença significativa entre os tratamentos (T1 e T2). Observou-se também que as amostras T2, T3, T4 e T5 (Tabela 4) foram significativamente iguais entre si quanto à prefência. Entretanto a amostra T1 (175 de soma das ordens) foi mais preferida e significativamente diferentes das amostras T3, T4 e T5 (soma das ordens de 131, 155 e 127), que foram menos preferidas (Tabela 4). Segundo Piana et al. (2004), a aplicação dos teste sensoriais do mel permite distinguir a origem botânica do mesmo, além de permitir a identificação e quantificação de certos defeitos, odores e sabores.

Tabela 4. Valores de soma das ordens de ordenação-preferência das amostras de mel com marca igual e floradas diferentes.

\begin{tabular}{cc}
\hline Tratamentos & Soma das ordens \\
\hline T1 & $175 \mathrm{a}$ \\
T2 & $162 \mathrm{ab}$ \\
T3 & $131 \mathrm{~b}$ \\
T4 & $155 \mathrm{~b}$ \\
T5 & $127 \mathrm{~b}$ \\
\hline
\end{tabular}

Legenda: $\mathrm{T} 1$ = flores silvestre; $\mathrm{T} 2=$ flores de eucalipto; $\mathrm{T} 3=$ flores de laranjeira; $\mathrm{T} 4=$ flores do sertão; $\mathrm{T} 5=$ flores de cipó de uva.

Médias na mesma coluna e com letras iguais não diferem $(p \leq 0,05)$ significativamente entre si, pelo teste de Tukey.

\section{CONCLUSÕES}

A maioria dos provadores: são do sexo feminino (59\%); consome mel (79\%); não sabe diferenciar o mel tradicional do orgânico (64\%); compraria mel orgânico (89\%); conhece (67\%), consome (75\%) e compraria (78\%) mel do produtor local; prefere comprar (67\%) e consumir (72\%) mel com selo do Serviço de Inspeção Federal. A maioria dos consumidores tem conhecimento sobre o mel, porém não costumam ler os rótulos dos produtos. Independente do tipo de florada todas as amostras de mel tiveram uma boa aceitação $(7,04$ a 7,86$)$ para todos os parâmetros avaliados e boa intenção de compra $(3,76$ a 4,14). O mel de florada silvestre foi mais preferido quando comparado aos de floradas de laranjeira, do sertão e de cipó de uva. Independente do tipo de florada todas as amostras obtiveram índice de aceitabilidade (IA) acima de $70 \%$. Portanto todas as amostras serão aceitas pelo público consumidor.

\section{AGRADECIMENTOS}

Ao Instituto Federal Goiano Campus Ceres pela bolsa PIBIC de Iniciação Científica.

\section{REFERÊNCIAS}

BARROS NETO, B.; SCARMINIO, I. S.; BRUNS, R. E. Como fazer experimentos: pesquisa e desenvolvimento na ciência e na indústria. Campinas: UNICAMP, 2003. 
BASTOS, D. H. M.; FRANCO, M. R. B.; DA SILVA, M. A. A. P.; JANZANTTI, N. S.; MARQUES, M.O.M. Composição de voláteis e perfil de aroma e sabor de méis de Eucalipto e laranja. Ciência e Tecnologia de Alimentos, v.22, n.2, p. 122-129, 2002. http://dx.doi.org/10.1590/S010120612002000200004 .

CAMPOS, G.; DELLA-MODESTA, R. C.; SILVA, T. J. P.; BAPTISTA, K. E.; GOMIDES, M. F.; GODOY, R. L. Classificação do mel em floral ou mel de melato. Ciência e Tecnologia de Alimentos, v. 23, n. 1, 2003. http://dx.doi.org/10.1590/S0101-20612003000100002.

FERREIRA, E. L., LENCIONI, C., BENASSI, M. T., BARTH, M. O., BASTOS, D. H. M.. Avaliação sensorial de mel de abelhas indígenas de diferentes localidades do Brasil. 2009. Disponível em: http://www.apacame.org.br/mensagemdoce/93/artigo3.htm. Acesso em: 05 Jun. 2016

GUYTON, A. C. Tratado de fisiologia médica. Rio de Janeiro: Elsevier, 2006.

IMPERATRIZ-FONSECA, V. L.; SARAIVA, A. M.; JONG, D. Bees as pollinators in Brazil: assessing the status and suggesting best practices. Ribeirão Preto: Holos, 2006.

LACERDA, J. J. J.; SANTOS, J. S.; SANTOS, S. A.; RODRIGUES, G. B.; SANTOS, M. L. P. Influência das características físico-químicas e composição elementar nas cores de méis produzidos pro Apis mellifera no sudoeste da Bahia utilizando análise multivariada. Química Nova, v. 33, n. 5, p.1022-1026, 2010. http://dx.doi.org/10.1590/S0100-40422010000500003.

MINIM, V. P. R. Análise sensorial: estudos com consumidores. Viçosa: Ed. UFV, 2010.

PIANA, M. L.; ODDO, L. P.; BENTABOL, A.; BRUNEAU, E.; BOGDANOV, S.; DECLERCK, C. G. Sensory analysis applied to honey: state of the art. Apidologie, v. 35, p. 26-37, 2004. https://doi.org/10.1051/apido:2004048.

R DEVELOPMENT CORE TEAM. R: a language and environment for statistical computing, reference index version 2.11 .1 (2010-05-31). Vienna: The R Foundation for Statistical Computing, Austria, 2010. Disponível em: https://cran.r-project.org/bin/windows/base/old/2.11.1/. Acesso em: 25 nov. 2010.

ROCHA, J. S.; BURITI, F. C. A.; SAAD, S. M. I. Condições de processamento e comercialização de queijo-de-minas frescal. Arquivo Brasileiro de Medicina Veterinária e Zootecnia, v.58, n.2, p.263272, 2006. http://dx.doi.org/10.1590/S0102-09352006000200016.

RODRIGUES, A. E.; SILVA, E. M. S.; BESERRA, E. M. F.; RODRIGUES, M. L. Análise físico-química de méis das abelhas Apis Mellifera e Melipona Scutellaris produzidos em duas regiões no Estado da Paraíba. Ciência Rural, v.35, n.5, p.1166-1171, 2005. http://dx.doi.org/10.1590/S010384782005000500028.

SANTOS, D. C.; OliveIRA, E. N. A.; JOABIS NOBRE MARTINS, J. N.; ALBUQUERQUe, E. M. B. Qualidade físico-química e microbiológica do mel de Apis mellifera comercializado na cidade de Russas, CE. Tecnologia \& Ciência Agropecuária, v.5, n.1, p.41-45, 2011. Disponível em: http://revistatca.pb.gov.br/edicoes/volume-05-2011/volume-5-numero-1-marco2011/tca08_qualidade_mel.pdf. Acesso em: 10 jun. 2016. 
SODRÉ, G. S.; CARVALho, C. A. L.; FOnSECA, A. A. O.; ALVES, R. M. O.; SOUZA, B. A. Perfil sensorial de amostras de méis de espécies de abelhas sem ferrão do Estado da Bahia. Magistra, v. 18, n. 4, p. 265-269, 2006.

SOUZA, D. A.; GRAMACHO, K. P.; CASTAGNINO, G. L. B. Produtividade de mel e comportamento defensivo como índices de melhoramento genético de abelhas africanizadas (Apis mellifera L.). Revista Brasileira de Saúde e Produção Animal, v.13, n.2, p 550-557, 2012. http://dx.doi.org/10.1590/S1519-99402012000200022.

TEIXEIRA, E.; MEINERT, E. M.; BARBETTA, P. A. Análise sensorial de alimentos. Florianópolis: UFSC, 1987. 